\title{
Delir in der Alterstraumatologie
}

\author{
Clemens Becker, Kerstin Bühl
}

\section{Zusammenfassung}

Die Alterstraumatologie gewinnt einen immer höheren Stellenwert in den unfallchirurgischen Abteilungen. Ohne diese Fallgruppen ist eine Abteilung auf Dauer wirtschaftlich nicht überlebensfähig. Entsprechend bedeutsam ist die angemessene Versorgung der älteren Patienten. Neben der hochwertigen unfallchirurgischen Behandlung gewinnt die geriatrische-internistische Behandlung eine größere Bedeutung. Die Prävention und Behandlung häufiger Komplikationen sollte rasch, zielgerichtet und evidenzbasiert erfolgen. Zu den häufigsten alterstraumatologischen Komplikationen gehört das Delir. Hierzu gibt es eine wachsende Anzahl an hochwertigen Publikationen. Delir gilt als teilweise verhinder- und therapierbar. Der Artikel fasst die Datenlage zusammen und gibt praktische Hinweise für den klinischen Alltag.

\section{Delirium in Geriatric Traumatology}

Geriatric traumatology is becoming more and more important in accident surgery departments. Without this group of cases such departments are not economically viable in the long term. An adequate care of the elderly patients is equally important. In addition to the high-value accident surgical management, geriatric medical treatment is also gaining a higher relevance. Prevention and treatment of the more frequent complications in this group should be rapid, targeted and evidence-based. Delirium is one of the most frequent complications among geriatric trauma patients. There is an increasing number of high-quality publications on this topic. Delirium is considered in part to be avoidable and to be curable. The present article summarises the currently available data and provides practical tips for the clinical routine.

\section{Einführung}

Ältere unfallchirurgische Patienten mit kognitiven Einschränkungen sind eine wachsende Herausforderung in nahezu allen traumatologischen Abteilungen im deutschsprachigen Raum. Die Abläufe im Rettungsdienst, in der Notaufnahme, auf den Intensivstationen und in der Routine der unfallchirurgischen Stationen sind hierauf meist nicht ausreichend vorbereitet. Häufig werden die Patienten als Belastung und nicht als wichtige Zielgruppe wahrgenommen, obwohl durch die Behandlung alterstraumatologischer Patienten bis zu 40\% und mehr der Erlöse der Abteilungen erwirtschaftet wer-

OP-JOURNAL 2014; 30: 40-45

(C) Georg Thieme Verlag KG Stuttgart · New York DOI http://dx.doi.org/10.1055/s-0034-1368264

berufe eine Übersicht zum Wissensstand des Themas zu geben. Dies beinhaltet die Bereiche Erfassung und Einschätzung (Assessment), Prävention und Therapie. Außerdem fasst der Artikel die wichtigsten evidenzbasierten Handlungsansätze zusammen. Evidenz muss in lokal adaptierte Vorgaben übertragen werden. One size does not fit all [3]. Dafür wird ein Beispiel eines „lokalen“ Behandlungspfads dargestellt. Weiterhin wird auf verschiedene „Best Practice“-Modelle im deutschsprachigen Raum verwiesen.

Bei jedem älteren Patienten sollte ein Screening auf das Vorliegen eines Delirs erfolgen.

\section{Definition, Phänomenologie und Diagnose des Delirs}

Ein Delir wird nach den gegenwärtig gültigen Diagnosekriterien (ICD-10 oder DSM-V) bei folgenden Symptomen diagnostiziert: akuter Beginn und fluktuierender Verlauf, Störung des Bewusstseins und der Aufmerksamkeit, Einschränkungen von Gedächtnis und Orientierung, psychomotorische Störungen (Erregtheit oder Apathie), Schlafstörungen sowie Nachweis einer organischen Grundlage. Die Abgrenzung zur Demenz kann dabei in der Akutsituation ohne Fremdanamnese schwierig sein und ist in Unkenntnis der Vorgeschichte (alleinlebender Patient) nicht immer möglich. Delirien sind bei Demenzpatienten besonders häufig. Die Fluktuation, psychomotorische Probleme und Schlafstörungen benötigen v.a. eine gute Pflegedokumentation und sind in der Regel allein ärztlich nicht ausreichend zu diagnostizieren. Die am weitesten verbreitete Skala zur Erfassung des Delirs ist die Confusion Assessment Method (CAM) (Abb. 1). Diese wurde ursprünglich in Yale in den USA entwickelt und wurde von der Arbeitsgruppe um C. Thomas ins Deutsche übersetzt. Die CAM wird seit Längerem in geriatrischen und alterstraumatologi- 


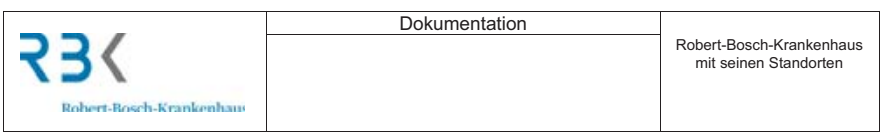

ET - Delirscreenning (CAM)

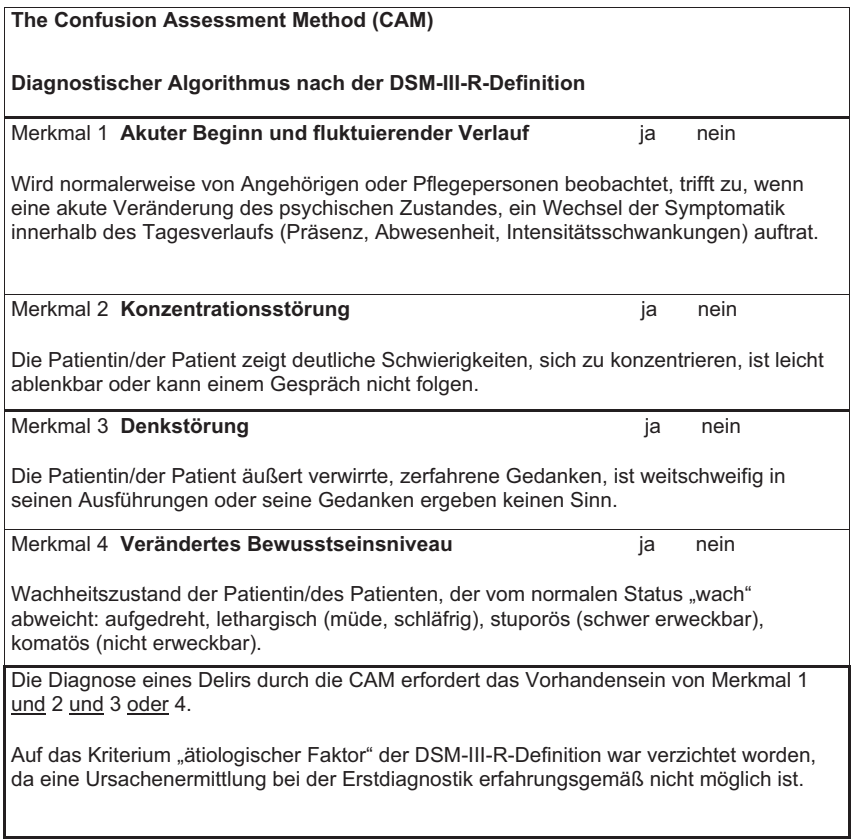

Verdacht auf Delir

ja nein

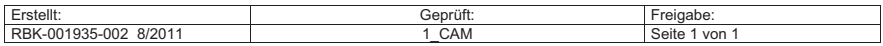

Abb. 1 ET-Delirscreening (CAM).

schen Zentren angewendet $[1,5]$. Hyperaktive Delirien werden in der Regel erkannt. Die Patienten „stören“ den Ablauf durch Unruhe und repetitives und anderes „herausforderndes“ Verhalten. Hypoaktive Delirpatienten werden häufiger „übersehen“, da sie meist den Ablauf der Station nicht behindern. Dabei ist die Prognose der hypoaktiven Delirformen besonders ungünstig. Die Gründe hierfür sind nicht klar. Es ist ratsam, sich mit der CAM und eventuellen Alternativen vertraut zu machen. Wichtig sind die Testgütekriterien und festzulegen, wer für die Durchführung verantwortlich ist. Mit der CAM lässt sich der Schweregrad eines Delirs nicht beschreiben. Hierfür sind andere Verfahren erforderlich.
Jede Abteilung sollte einen Behandlungspfad für die Prävention und Behandlung von Delirpatienten erstellen (Abb. 2).

\section{Inzidenz und Prävalenz}

Derzeit leiden 7-8\% der älteren Menschen (hier: Personen >64 Jahre) in Deutschland unter einer Demenz als häufigstem Risikofaktor eines Delirs. Demenzkranke haben ein 3-fach erhöhtes Risiko, eine sturzbedingte Fraktur zu erleiden. Diese Gruppe hat auch das höchste Risiko, während eines Krankenhausaufenthalts ein Delir zu erleiden. Daneben ist damit zu rechnen, dass viele äl- tere Patienten während eines unfallchirurgischen Aufenthalts ein Delir entwickeln werden, ohne vorher an einer Demenz zu leiden. Bei angemessener Delirdiagnostik bedeutet dies, dass mehr als $40 \%$ der Patienten mit proximalen Femurfrakturen (PFF) ein Delir entwickeln werden. Dabei ist das Ausmaß der vorbestehenden Neurodegeneration und vaskulären Hirnschäden entscheidend. Derzeit kommen in Deutschland 20\% der ca. 120000 PFF-Patienten [10] aus einer Pflegeeinrichtung, 30\% werden vor der PFF bereits zu Hause gepflegt. Nur die Hälfte der Betroffenen hatte prästationär noch keinen formellen Pflegebedarf [12]. Für andere Frakturtypen sind die Zahlen bisher weniger gut untersucht [9]. Delir bei PFF ist mit höheren Komplikationsraten, höherer Mortalität und längeren Aufenthaltsdauern verbunden [7]. Es ist davon auszugehen, dass die Inzidenz bei Becken- und bei einem Teil der hochaltrigen Wirbelkörper- und Oberarmfrakturen ähnlich hoch ist. Bisher wenig untersucht ist die Rolle einer begleitenden traumatischen Hirnverletzung bei PFF. In einer wegweisenden Untersuchung mit Videoanalyse von Stürzen, die 2013 in Kanada veröffentlicht wurde, konnte gezeigt werden, dass es bei mehr als der Hälfte der Stürze von Heimbewohnern zu einem „Schädelhirntrauma“ kam [13]. Dies bedarf der dringenden weiteren Untersuchung.

Die Priorisierung dieser Behandlungsgrundsätze ist Leitungsaufgabe.

\section{Risikofaktoren}

Neben der Demenz gibt es zahlreiche weitere Risikofaktoren, die das Auftreten eines Delirs verursachen können [2]. Zu den häufigsten Risikofaktoren gehören Infektionen (Harn- und Atemwege), Überlaufblase, Nierenversagen, Elektrolytverschiebungen wie Hyponatriämie, nicht erkannte oder unzureichend behandelte Schmerzen und anticholinerg wirkende Medikamente wie Neuroleptika (z. B. Promethazin/Atosil oder Levomepromazin/Neurocil) und ParkinsonMedikamente. Daneben können Umgebungsfaktoren wie ein Ortswechsel (Notaufnahme, Intensivstation, UC-Station), häufiger Wechsel der behandelnden Personen, hoher Lärmpegel, sensorische Deprivation ein Delir triggern bzw. den Schweregrad des Delirs beeinflussen. Nicht zuletzt ist auf einen vorbestehenden Substanzmissbrauch (Alkohol oder Benzodiazepine) zu achten. 


\section{Fallbericht}

Eine 88 Jahre alte Patientin wird mit Verdacht auf eine Femurfraktur an einem Samstagvormittag eingewiesen. Sie hat bisher allein gelebt. Die Liegezeit in der Wohnung lag vermutlich bei mehreren Stunden. Die Nachbarn hatten gehört, wie die Patientin laut gerufen hat. Zur medizinischen Vorgeschichte ist wenig bekannt. Die Medikamente wurden vom Rettungsdienst nicht mitgebracht. Kinder sind soweit bekannt nicht vorhanden. Der Hausarzt ist am Wochenende nicht erreichbar. Die Patientin ist örtlich orientiert. Sie ist zeitlich etwas unscharf orientiert (Jahresangabe des Vorjahrs, Alter und Geburtsjahr sind korrekt). Die medizinische Anamnese ist erschwert. Die Patientin ist schwerhörig. Ein Hörgerät wurde vom Rettungssanitäter nicht mitgebracht. Die Röntgenuntersuchung bestätigt die Verdachtsdiagnose (pertrochantäre Fraktur). Die Laboruntersuchungen und das Ruhe-EKG sind bis auf eine Kreatininclearance von $40 \mathrm{ml} /$ min unauffällig. Das präoperative anästhesiologische Konsil ergibt keine Einwände gegen eine zügige Operation.

\section{Empfehlungen zum Ablauf der Behandlung}

\section{Vorstationäres Vorgehen}

Bereits vor der stationären Einweisung sollten erste Behandlungsmaßnahmen eingeleitet werden. Bei vielen häuslichen Stürzen handelt es sich um ein Langliegetrauma, bevor der Hausarzt oder die Rettungsleitstelle verständigt werden konnte. Entsprechend wichtig ist die Gabe von parenteraler Flüssigkeit und Analgetika. Im Rettungswagen sollte der kognitive Status vorgeklärt werden. Zumindest sollte klar sein, ob der Patient/ die Patientin (im Weiteren die Patientin) zeitlich, örtlich und situativ orientiert ist. Dies sollte protokollarisch festgehalten werden.

\section{Notfallambulanz}

Die ärztliche Diagnostik sollte möglichst zügig durchgeführt werden. Bei einem etablierten Triagesystem sollte die Patientin als Risikopatientin klassifiziert werden. Die Pflegeaufnahme sollte ein kognitives Screening beinhalten (s.u.). Vor allem bei Patienten mit bekannten oder vermuteten kognitiven Problemen sollten lange Wartezeiten unbedingt vermieden werden. Bei Personen mit unklarem Sturzhergang, v.a. Bewohnern

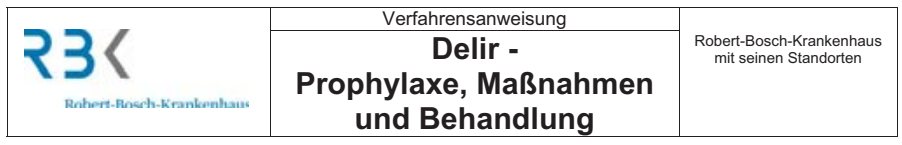

Änderungshinweise:

1 Zweck

Regelt die Vorgehensweise während des orthopädisch-unfallchirurgisch akutstationären Akutaufenthaltes bei Patienten mit deliranten Symptomen

\section{Geltungsbereich}

ZAT Verantwortung / Zuständigkeiten

Ärzte Orthopädie und Unfallchirurgie

Assistenzarzt Zentrum für Alterstraumatologie (ZAT)

Pflegemitarbeiter Orthopädie und Unfallchirurgie

Ergo-/Physiotherapeuten Orthopädie und Unfallchirurgie

Mitarbeiter des Entlassmanagements Orthopädie und Unfallchirurgie

\section{Verfahren / Vorgehensweise}

A. Basisdiagnostik und-maßnahmen durch OUCH Ärzte NAZ und ZAT

o. Empathischer Umgangston und konfliktvermeidendes Verhalten

o. Klinische Anamnese, ggf. Fremdanamese

o. Körperliche Untersuchung

. Medikamentenanamnese

. Erfassung von Risikofaktoren für die Entstehung eines Delirs (Hinweis auf kognitive Einschränkungen? Psychopharmaka?, Hör- oder Sehstörungen?)

o. Vermeidung von Patientenverlegungen auf andere Stationen als $1 \mathrm{~F}$ (abgesehen von Überwachungsstationen aus medizinischen Gründen)

o. Gute Schmerzbehandlung

o. Vermeiden und Erkennen von Infektionen

o. Ausgleich von Flüssigkeitsverlusten und Elektrolytstörungen

o. Anpassung der Medikation

o. psychiatrisches Konsil und/oder RS mit Geriater

o. Anordnung frühzeitiger Mobilisierung und Anforderung von Physiotherapie

o. bei Bedarf Kontaktaufnahme mit den Angehörigen

o. Information der Angehörigen zum Thema Delir und Ermutigung zu Besuchen

o. ggf. CCT zum Ausschluss einer Blutung

o. Tägliche Überprüfung der psychiatrischen Medikation bei den Visiten

o. Dokumentation im ZAT-Verlaufsbericht (gaplt)

B. Labordiagnostik

ZAT-Labor (beinhaltet Vit. B12, crP, Elektrolyte, TSH, Retentionswerte)

C. Aufgaben und Maßnahmen der Pflegemitarbeiter des ZAT

o. Empathischer Umgangston und konfliktvermeidendes Verhalten

o. feste Bezugsperson anstreben

o. Vermeidung von Verlegungen auf andere Stationen oder in andere Zimmer

o. DK frühzeitig entfernen

o. Frühmobilisierung durch aktivierende Pflege

\begin{tabular}{|l|l|}
\hline RBK-002120-001 10/2012 De & Der
\end{tabular} Seite 1 von 2

Abb. 2a Behandlungspfad Delir.

von Pflegeheimen, besteht ein hoher diagnostischer Verdacht auf ein begleitendes Schädel-Hirn-Trauma [13]. Nach Durchführung der klinischen und radiologischen Untersuchung sollte umgehend über den geplanten OP-Zeitpunkt entschieden werden. In Abhängigkeit der logistischen Möglichkeiten erfolgt die Weiterversorgung auf der UC-Station. Dort erfolgt die weitere präoperative Diagnostik einschließlich der anästhesiologischen und ggf. geriatrischen Risikostratifizierung. Bei Langliegetraumen - v. a. bei Männern - muss eine Überlaufblase ausgeschlossen werden. Eine weitere wichtige Screeningmaßnahme ist der periphere Pulsstatus (pAVK und Dekubitusrisiko).

\section{Präoperative Behandlung} auf der UC-Station

Je nach geschätzter präoperativer Wartezeit (Kapazität) muss der Umgang mit den Patienten ähnlich dem mit anderen Hochrisikopatienten gestaltet werden. Alle Mitarbeiter sollten sich persönlich und wiederholt mit Namen und zugehöriger Rolle vorstellen. Immer wieder sollte der Ort der Behandlung, der Grund der Einweisung und das geplante Vorgehen ruhig und höflich genannt werden. Redundanz erhöht die Handlungssicherheit. Zeitliche, örtliche und situative Unsicherheiten können von den Mitarbeitern ausgeglichen werden. Besonders ist auf sensorische Einschränkungen $\mathrm{zu}$ 


\begin{tabular}{|c|c|c|}
\hline & Verfahrensanweisung & \\
\hline 231 & $\begin{array}{c}\text { Delir - } \\
\text { Prophylaxe, Maßnahmen } \\
\text { und Behandlung }\end{array}$ & $\begin{array}{l}\text { Robert-Bosch-Krankenhaus } \\
\text { mit seinen Standorten }\end{array}$ \\
\hline
\end{tabular}

o. Information an Betreuungsassistenten

-> Gruppenbetreuung vormittags im Aufenthaltsraum mit gemeinsamen Aktivitäten und Einnahme der Mahlzeiten

o. Förderung eines normalen Tag/Nachtrhythmus mit entsprechenden Lichtverhältnissen und angepasster Umgebungslautstärke

o. Überwachung der Nahrungs- und Flüssigkeitszufuhr

o. Erfassung von Schmerzen

o. Einbeziehung der Angehörigen

D. Maßnahmen und Aufgaben der Therapeuten

o. Empathischer Umgangston und konfliktvermeidendes Verhalten

o. Screening auf Delirsymptome mittels CAM durch die Ergotherapeuten

(Aktuell nur bei Patienten, die eine geriatrische Komplexbehandlung erhalten, möglich. Zusätzlich wird ein GDS-5 und BOMCT erstellt)

o. Frühmobilisierung

o. Kognitive Aktivierung und Selbsthilfetraining durch die Ergotherapeuten

E. Aufgaben und Maßnahmen der Mitarbeiter des Entlassmanagements

o. Empathischer Umgangston und konfliktvermeidendes Verhalten

o. soziales Assessment

0. ggf. Kontaktaufnahme mit den Angehörigen

F. Medikamentöse Behandlung

Falls nicht medikamentöse Maßnahmen nicht ausreichen und/oder der Patient sich ode andere gefährdet:

o. Psychiatrisches Konsi

o. Melperon $25 \mathrm{mg}$ (entspr. $5 \mathrm{ml}$ Liqu.) abends, ggf. schrittweise Steigerung auf bis zu 100 (200) $\mathrm{mg} / \mathrm{Tag}$ verteilt auf mehrere Einzeldosen

o. Bei halluzinatorischen oder wahnhaften Verkennungen 0.5 bis $1 \mathrm{mg}$ Haloperido (entspricht $5-10$ Tropfen) als Initialdosis

Cave bei M. Parkinson, Parkinsonsyndrom oder Lewy-körperchen Demenz, dann RS mit Neurologen

F Arztbrief

Übernahme der Diagnose hypoaktives oder hyperaktives Delir in den Arztbrief mit ggf. Empfehlung der weiteren (medikamentösen/nicht medikamentösen) Behandlung

\section{Mitgeltende Unterlagen}

AK Versorgungsstrukturen der AG Alterstraumatologie der DGU / AK Versorgungsstrukturen der AG Alterstraumatologie der DGU /DGG, Abschließende Überarbeitung Version 2.1:

04.05.12/13.11.12 Dr. Riem, Dr. Meyjohann, Dr. Leischker

Arbeitsgemeinschaft der Wissenschaftlichen Medizinischen Fachgesellschaften (AWMF):

S3-Leitlinie Analgesie, Sedierung und Delirmanagement in der Intensivmedizin (2009)

S3-Leitlinie Demenzen (2009)

National Institute for Health and Clinical Excellence (NICE):

Delirium: Diagnosis, Prevention and Management (July 2010)

5 Dokumentation

Optiplan und ZAT-Verlaufsbericht (gaplt)

\begin{tabular}{|l|l|l|}
\hline RBK-002120-001 10/2012 & Delir & Seite 2 von 2 \\
\hline
\end{tabular}

Abb. 2b Behandlungspfad Delir.

achten. Nicht selten werden schwerhörige Patienten als dement fehlklassifiziert oder visuelle Einschränkungen wie Makuladegeneration nicht erkannt.

Ein präoperativer kognitiver Status ist für die Verlaufsbeurteilung unerlässlich. Es sollte klar sein, wer diesen erhebt (ggf. Unfallchirurg, Anästhesist, Geriater, Pflege). Ein Screening ist in der Regel zunächst ausreichend. Mögliche Instrumente sind Mini-Cog, Short Orientation Memory Scale, Abbreviated Mental Test Score. Assessment-Instrumente wie der MMST sind meist zu zeitaufwendig und daher nicht sinnvoll.
Bei kognitiv eingeschränkten Personen mit Schmerzen ist eine VAS-Bewertung (Visuelle Analogskala) meist nicht möglich. Hier muss abteilungsintern eine Alternative gesucht werden. Ein mögliches Verfahren ist die NRS. Bei Patientinnen mit fortgeschrittener Demenz sind Verfahren wie die Pain-AD-Skala oder die BEST-Skala geeignet.

Jede Abteilung sollte einen Behandlungspfad entwickeln, der auf die örtlichen Gegebenheiten eingeht.

\section{Fallbericht - Fortsetzung}

Das präoperative anästhesiologische Konsil ergab keine anästhesiologischen
Einwände gegen eine zügige Operation. Die Operation wurde für den folgenden Morgen terminiert. Geplant wurde eine Vollnarkose. Unfallchirurgisch ist eine intramedulläre Versorgung vorgesehen. Die ergänzend durchgeführten Untersuchungen erbrachten keinen Hinweis auf eine pAVK. Der Hautstatus war intakt. Der Mini-Cog zeigte eine falsch gezeichnete Uhr als Hinweis auf eine visuell-konstruktive Einschränkung. Die Patientin zeigte in der Nacht Durchschlafstörungen, war aber weiterhin zur Person orientiert. Zeitlich und örtlich war sie am Morgen unscharf orientiert (Verwechslung des Jahres und falsche Angabe des Krankenhauses). Die Schmerztherapie erfolgte mit WHO-Schema Stufe II. Es erfolgte eine perioperative Antibiotikaprophylaxe und Heparingabe. Die unfallchirurgische Versorgung verlief technisch ohne besondere Vorkommnisse. Die postoperative Kontrolle zeigte eine Anämie (Hb 9,0 g/dl). Die Kreatininclearance lag bei $34 \mathrm{ml} / \mathrm{min}$. Die Elektrolyte waren ausgeglichen. Die Patientin wurde postoperativ überwacht und anschließend auf der alterstraumatologischen Station weiterversorgt. Der Troponinspiegel war grenzwertig erhöht. Das Kontroll-EKG zeigte keine Auffälligkeiten.

\section{Perioperatives Management}

Es besteht Konsens, dass ein multifaktorieller Ansatz wirksam den Schweregrad bzw. die Inzidenz des Delirs beeinflussen kann [4]. Ob Einzelmaßnahmen oder welche Einzelmaßnahmen Einfluss auf den Schweregrad bzw. die Inzidenz des Delirs haben, ist bislang unklar. Derzeit sollten die allgemeingültigen Leitlinien in der Behandlung von Hüftfrakturpatienten eingehalten werden (Blue Book oder z.B. Scottish Intercollegiate Guideline Network - SIGN). Hierzu gehören eine frühzeitige Operation, eine frühzeitige postoperative Mobilisation, eine angemessene leitliniengerechte Schmerztherapie, ein bilanzierter Flüssigkeitshaushalt, die Vermeidung delirogener Medikamente, ein hoher diagnostischer Verdacht auf Blasenentleerungsstörungen, eine Überwachung der Nierenfunktion und des Elektrolythaushalts. Ob Regional- oder Vollnarkose günstiger sind, ist nicht geklärt. Auch für Patienten mit kardialer Vorgeschichte gibt es keine Evidenz für bessere Ergebnisse einer liberalen Transfusionsindikation, wenn der $\mathrm{Hb}$-Wert mehr als $8 \mathrm{~g} / \mathrm{dl}$ beträgt. 


\section{Pharmakologische Delirprävention}

Seit mehreren Jahren wurden verschiedene kleinere RCTs mit klassischen und atypischen Neuroleptika zur Delirprävention durchgeführt [8]. Die Hypothese der Phase-II-Studien war eine Reduktion der Delirschwere bzw. die Inzidenz der Delirien zu reduzieren. Hierbei wurden Hochrisikopatienten (Rezidivprophylaxe) oder Patienten mit moderaterem Risikoprofil eingeschlossen. Die verwendeten Substanzen waren Haloperidol und atypische Substanzen wie Quetiapin/Seroquel. Einzelne Studien waren erfolgreich, andere zeigten keine positiven Ergebnisse. Bislang konnte somit kein eindeutiger Nutzen belegt werden. Auch Versuche mit Antidementiva waren bislang ebenfalls erfolglos [6]. Eine begründete Vorbehandlung mit Neuroleptika sollte fortgeführt werden. Dies gilt ebenso für andere zentralwirksame Substanzen wie Parkinson-Medikamente, Antidepressiva und Benzodiazepine.

\section{Wach-/Intensivstation}

Beim Auftreten von Kreislaufproblemen, erheblichem Transfusionsbedarf oder Organversagen wird häufiger eine meist kurzfristige Betreuung auf einer Überwachungsstation oder Intensivstation erforderlich sein. Derzeit liegt die postoperative Mortalität (7. bzw. 14. postoperativer Tag) in den meisten Ländern bei 5-10\%. In Ländern mit konsequenten und ausreichend finanzierten Qualitätsverbesserungsprozessen wie in England konnte ein Rückgang der postoperativen Mortalität gezeigt werden. In Deutschland fehlen bisher ein entsprechender Nachweis bzw. die dafür erforderlichen Untersuchungen. Die postoperative Behandlung und Überwachung auf den genannten Stationen bietet medizinische Vorteile, hat aber auch aus Sicht der Delirprävention erhebliche Risiken. Auf den Intensivstationen herrschen oft eine erhebliche Unruhe und Lärmbelästigung. Die räumliche, zeitliche und situative Orientierung ist erschwert. Hierdurch kann ein Delir erst ausgelöst werden. Daher sollte kritisch geprüft werden, welche Vor- und Nachteile eine Behandlung dort beinhaltet. Für die ICU und IMC-Einheiten wurden angepasste Skalen der CAM entwickelt (CAM ICU).

\section{Fallbericht - Fortsetzung}

In der 1. postoperativen Nacht war die Patientin zunehmend unruhig und versuchte aufzustehen, was von der Zim- mernachbarin rechtzeitig signalisiert wurde. Vom Dienstarzt wurde ein niederpotentes Neuroleptikum angeordnet, dass vom AID für ältere Patienten als bedingt geeignet klassifiziert wurde (Melperon). Die am darauffolgenden Morgen durchgeführte Restharnkontrolle zeigte einen Wert von $250 \mathrm{ml}$. Eine Frührehabilitation wurde am 2. postoperativen Tag begonnen. Die entsprechenden Assessments wurden physiotherapeutisch (Trunk Control Test, Rivermead-Mobilitätsskala, Standpositionen) und ergotherapeutisch (AMTS, häuslich-soziale Versorgung) durchgeführt. Es bestand zum ersten Mal die Möglichkeit, Kontakt mit dem Hausarzt und wohnortfernen Verwandten aufzunehmen. Die ambulante Weiterversorgung im unmittelbaren Anschluss an die Unfallchirurgie wurde von den Teammitgliedern als kritisch bewertet. Bei der 1. Teambesprechung der Frührehabilitation wurde beschlossen, eine Anschlussbehandlung in einer stationären geriatrischen Rehabilitationseinrichtung durch die Case Managerin zu beantragen. Die Nierenfunktion verschlechterte sich in Folge einer Exsikkose. Eine postrenale Ursache konnte ausgeschlossen werden. Die Schmerzen waren unter Stufe-II-Medikation ausreichend kontrolliert. Die postoperative Röntgenkontrolle war zufriedenstellend. Der Wund- und Hautstatus war regelrecht. Am 3. und 4. postoperativen Tag war die Patientin sehr still, klagte nicht. Die Orientierung war schlechter. Die Trinkmenge lag unter einem Liter pro Tag. Eine parenterale Flüssigkeitsgabe wurde eingeleitet. Die Patientin entfernte mehrfach die Infusion. Die festangesetzte Neuroleptikatherapie wurde beendet. Die Patientin wurde in ein intensiviertes pflegerisches Betreuungsprogramm integriert. Dies beinhaltete eine 8-h-Betreuung mit Pflegeassistenz. Dadurch konnte eine Verbesserung der Flüssigkeitszufuhr erreicht werden. Die Nierenfunktion verbesserte sich.

\section{Erweitertes postoperatives Management}

Eine möglichst frühzeitige Mobilisation und angemessene Schmerztherapie sind essenziell.

Falls der Schweregrad des Delirs erfasst werden soll oder muss, eignen sich Assessment-Verfahren wie die Delirium Rating Scale (DRS) oder die Delirium Severity Scale (DSS). Hierbei muss bedacht werden, dass diese Verfahren einen höheren Zeit-, Schulungs- und damit Personalaufwand bedeuten. In dieser Zeit ist eine geriatrische Mitbetreuung essenziell. Dies bedeutet eine geregelte und wertschätzende Zusammenarbeit. Konsil- und Liaisondienste sind hierfür ungeeignet. Die Teamleitung in der Komplexbehandlung obliegt dem Geriater. Die Leitung der Unfallchirurgie ist damit aber nicht infrage gestellt. Ob Frühverlegungen in die Geriatrie (z.B. ab dem 3. postoperativen Tag) einer unfallchirurgischen Behandlung mit Integration geriatrischer Kompetenz überlegen ist, ist derzeit unklar. In dem umfangreichen $(n=400)$, randomisiert kontrollierten Trondheim Hip Fracture Trial in Norwegen wurde dies nachgewiesen [11]. Allerdings kann dies nicht ohne weiteres auf die deutschen Strukturen übertragen werden. Hier sind Beobachtungs- und Interventionsstudien im deutschsprachigen Raum gefordert.

\section{Fallbericht - Fortsetzung}

Nach Genehmigung der Krankenkasse wurde die weiterführende Behandlung in der geriatrischen Rehabilitation fortgesetzt. Medizinische Inhalte dort war die Untersuchung der Knochendichte, Harninkontinenz, Mangelernährung, kognitive Testung und zerebrale Bildgebung, Visusprüfung, Überprüfung der Medikation (Medication Review), sekundäre Sturzprävention einschließlich eines diagnostischen Hausbesuchs geplant und durchgeführt. Diese Komponenten werden an dieser Stelle nicht weiter ausgeführt. Der kognitive Status war leicht gebessert. Die Patientin war in der Lage, bei den Therapien mitzuarbeiten.

\section{Best-Practice-Beispiele}

Die wichtigsten Forschungsarbeiten zum Thema Delir wurden an der Universitäsklinik in Yale $[3,4]$ erarbeitet und publiziert. Dort wurde das HELP-Programm entwickelt (Hospital Elderly Life Program). Andere international wichtige Orte sind in Schweden die Universitätsklinik Umea und in der Schweiz die Universitätsklinik Basel. Wichtige Leuchtturmprojekte in Deutschland sind in Bielefeld (PD Dr. Thomas) und Münster zu finden (Dr. Gurlit). Es gibt einen internationalen Konsens, dass die Inzidenz und der Schweregrad der perioperativen Delirien beeinflussbar sind. Hierfür ist ein interdisziplinäres und interprofessionelles Vorgehen unabdingbar. 


\section{Rollen}

Die Hierarchien und das Rollenverständnis ist in Deutschland weiterhin strukturell konservativ. Dies ist im Zusammenhang mit der Therapie und Prävention des Delirs in der Alterstraumatologie hinderlich. Dies betrifft sowohl die $\mathrm{Zu}$ sammenarbeit der ärztlichen Spezialitäten als auch die Zusammenarbeit mit den nicht ärztlichen Berufsgruppen. Im internationalen Vergleich besteht hier ein erheblicher Nachholbedarf. Die Rollen und Verantwortung sollten in der abteilungsspezifischen Diskussion festgehalten werden.

\section{Forschungsfelder}

Zur Versorgung anderer Frakturen im Bereich des Beckens, Wirbelsäule und Humerus gibt es wenig bis keine Literatur. Dies gilt auch international. Es ist durchaus vorstellbar, dass bestimmte Frakturtypen wie komplexe Beckenfrakturen noch häufiger mit den genannten Problemen konfrontiert sind, selbst wenn diese überwiegend konservativ behandelt werden. Aus geriatrischer Sicht ist es unbefriedigend, dass nach wie vor ungeklärt ist, welche Anästhesieformen ein höheres oder geringeres Risiko eines Delirs haben. Hier hat in den letzten 20 Jahren praktisch keine Forschung stattgefunden. Grundsätzlich ist es zu untersuchen, ob eine pharmakologische Delirprävention möglich ist. Eine offene Frage bleibt eine bessere Risikostratifizierung. Die Einzelmaßnahmen sollten weiterentwickelt und faktoriell getestet werden.

\section{Zusammenfassung und Ausblick}

In den letzten Jahren hat eine sehr positive Entwicklung in der Unfallchirurgie stattgefunden. Die Alterstraumatologie wird von fast allen Abteilungen nicht mehr als notwendiges Übel betrachtet, sondern es wird eine intensive Auseinandersetzung mit dem Thema gesucht. Dies schließt den manchmal kontroversen Dialog mit den Nachbardisziplinen ein. Nur so kann ein Erkenntnisgewinn gelingen. Die zuletzt festgefahrene Debatte um die Auditierung und Zertifizierung der Alterstraumatologie zeigt das große Interesse. Es zeigt sich aber auch, dass lobbyistische Partikularinteressen in eine Sackgasse führen. Die Alterstraumatologie in Deutschland ist derzeit unterfinanziert. Die Erlöse für die Frakturversorgung sind in der unteren Quartile im Vergleich mit vergleichbaren OECDLändern. Hier gibt es Handlungsbedarf. Aus Sicht der Delirprävention und Delirtherapie ist die gemeinsame Behandlung der Unfallchirurgie, Geriatrie und Anästhesie ein Muss und kein Kann. Delir ist eine der teuersten Komplikationen. Das Auftreten eines Delirs erhöht den Behandlungsaufwand erheblich. Delir ist aber oft verhinderbar bzw. der Schweregrad beeinflussbar. Die Behandlung muss gemäß der GCP-Prinzipien durchgeführt werden. Das Team muss in den delirspezifischen Aspekten geschult werden. Das ist nicht umsonst, trägt aber mittelfristig Früchte. Ohne den erklärten sichtbaren Willen der Führungskräfte sind diese Anstrengungen zum Scheitern verurteilt.

\section{Literatur}

${ }^{1}$ Hestermann U, Backenstrass M, Gekle I et al. Validation of a German version of the Confusion Assessment Method for delirium detection in a sample of acute geriatric patients with a high prevalence of dementia. Psychopathology 2009; 42: 270-276

2 Fong TG, Tulebaev SR, Inouye SK. Delirium in elderly adults: diagnosis, prevention and treatment. Nat Rev Neurol 2009; 5: 210-220

${ }^{3}$ Inouye SK. Delirium in older persons. N Engl J Med 2006; 354: 1157-1165
${ }^{4}$ Inouye SK, Bogardus jr. ST, Charpentier PA et al. A multicomponent intervention to prevent delirium in hospitalized older patients. N Engl J Med 1999; 340: 669-676

${ }^{5}$ Wei LA, Fearing MA, Sternberg EJ et al. The Confusion Assessment Method: a systematic review of current usage. J Am Geriatr Soc 2008; 56: 823-830

${ }^{6}$ Marcantonio ER, Palihnich K, Appleton P et al. Pilot randomized trial of donepezil hydrochloride for delirium after hip fracture. J Am Geriatr Soc 2011; 59 (Suppl. 2): S282-S288

7 Marcantonio ER, Flacker JM, Michaels $M$ et al. Delirium is independently associated with poor functional recovery after hip fracture. J Am Geriatr Soc 2000; 48: 618-624

8 Kalisvaart KJ, de Jonghe JF, Bogaards MJ et al. Haloperidol prophylaxis for elderly hip-surgery patients at risk for delirium: a randomized placebo-controlled study. J Am Geriatr Soc 2005; 53: 1658-1666

${ }^{9}$ Bleibler F, Konnopka A, Benzinger P et al. The health burden and costs of incident fractures attributable to osteoporosis from 2010 to 2050 in Germany-a demographic simulation model. Osteoporos Int 2013; 24: 835-847

${ }_{10}$ Icks A, Arend W, Becker C et al. Incidence of hip fractures in Germany, 1995-2010. Arch Osteoporos 2013; 8: 140

11 Saltvedt I, Prestmo A, Einarsen E et al. Development and delivery of patient treatment in the Trondheim Hip Fracture Trial. A new geriatric in-hospital pathway for elderly patients with hip fracture. BMC Res Notes 2012; 5: 355

12 Rapp K, Becker C, Cameron ID et al. Femoral fracture rates in people with and without disability. Age Ageing 2012; 41: 653-658

13 Schonnop R, Yang Y, Feldman $F$ et al. Prevalence of and factors associated with head impact during falls in older adults in long-term care. CMAJ 2013; 185: E803-E810

Prof. Dr. med. Clemens Becker Chefarzt

Dr. med. Kerstin Bühl

Oberärztin

Robert-Bosch-Krankenhaus Abteilung Geriatrie und Klinik für Geriatrische Rehabilitation Auerbachstraße 110 70376 Stuttgart

clemens.becker@rbk.de 